\title{
Specialties Performing Paracentesis Procedures at University Hospitals: Implications for Training and Certification
}

\author{
Jeffrey H. Barsuk, MD, MS ${ }^{1 *}$, Joe Feinglass, PhD, Sarah E. Kozmic, BS¹, Samuel F. Hohmann, PhD², \\ Daniel Ganger, $\mathrm{MD}^{3}$, Diane B. Wayne, MD'
}

${ }^{1}$ Department of Medicine, Northwestern University Feinberg School of Medicine, Chicago, Illinois; '2University HealthSystem Consortium, Chicago, Illinois; ' Division of Hepatology, Northwestern University Feinberg School of Medicine, Chicago, Illinois.

BACKGROUND: Paracentesis procedure competency is not required for internal medicine or family medicine board certification, and national data show these procedures are increasingly referred to interventional radiology (IR). However, practice patterns at university hospitals are less clear.

OBJECTIVE: To evaluate which specialties perform paracentesis procedures at university hospitals, compare characteristics of patients within each specialty, and evaluate length of stay (LOS) and hospital costs.

DESIGN, SETTING, PATIENTS: Observational administrative database review of patients with liver disease who underwent paracentesis procedures in hospitals participating in the University HealthSystem Consortium (UHC) Database from January 2010 through December 2012. UHC is an alliance of 120 academic medical centers and their 290 affiliated hospitals.

EXPOSURE: Patients with liver disease who underwent inpatient paracentesis procedures.

MEASUREMENTS: We compared characteristics of patients who underwent paracentesis procedures by physician specialty, modeling the effects of patient characteris- tics on the likelihood of IR referral. We also analyzed LOS and hospital costs among patients with a $>20 \%$ predicted probability of IR referral.

RESULTS: There were 97,577 paracentesis procedures performed during 70,862 hospital stays in 204 hospitals. IR performed $29 \%$ of paracenteses versus $49 \%$ by medicine and medicine subspecialties including gastroenterology/hepatology. Patients who were female, obese, and those with lower severity of illness were more likely to be referred to IR. Patients with a medicine or gastroenterology/hepatology paracentesis had a similar LOS compared to IR. Hospital costs were an estimated as $\$ 1308$ less for medicine and $\$ 803$ less for gastroenterology/hepatology compared to admissions with IR procedures (both $P=0.0001$ ).

CONCLUSIONS: Internal medicine- and family medicinetrained clinicians frequently perform paracentesis procedures on complex inpatients but are not currently required to be competent in the procedure. Increasing bedside paracentesis procedures may reduce healthcare costs. Journal of Hospital Medicine 2014;9:162-168. (C) 2014 Society of Hospital Medicine
Cirrhosis affects up to $3 \%$ of the population and is 1 of the 10 most common causes of death in the United States. ${ }^{1-4}$ Paracentesis procedures are frequently performed in patients with liver disease and ascites for diagnostic and/or therapeutic purposes. These procedures can be performed safely by trained clinicians at the bedside or referred to interventional radiology (IR). ${ }^{2-4}$

National practice patterns show that paracentesis procedures are increasingly referred to IR rather than performed at the bedside by internal medicine or gastroenterology clinicians. ${ }^{5-7}$ In fact, a recent study of Medicare beneficiaries showed that inpatient and outpatient paracentesis procedures performed by radiol-

\footnotetext{
*Address for correspondence and reprint requests: Jeffrey $\mathrm{H}$. Barsuk, MD, Division of Hospital Medicine, 211 E. Ontario St., Suite 717, Chicago, IL 60611; Telephone: 312-926-3680; Fax: 312-926-4588;

E-mail: jbarsuk@nmh.org

Additional Supporting Information may be found in the online version of this article.

Received: October 30, 2013; Revised: December 22, 2013; Accepted: December 23, 2013

2014 Society of Hospital Medicine DOI 10.1002/jhm.2153

Published online in Wiley Online Library (Wileyonlinelibrary.com).
}

ogists increased by $964 \%$ from 1993 to 2008 . $^{7}$ Reasons for the decline in bedside procedures include the increased availability of IR, lack of sufficient reimbursement, and the time required to perform paracentesis procedures. ${ }^{5-8}$ Surveys of internal medicine and family medicine residents and gastroenterology fellows show trainees often lack the confidence and experience needed to perform the procedure safely. ${ }^{9-11}$ Additionally, many clinicians do not have expertise with ultrasound use and may not have access to necessary equipment.

Inconsistent certification requirements may also impact the competence and experience of physicians to perform paracentesis procedures. Internal medicine residents are no longer required by the American Board of Internal Medicine (ABIM) to demonstrate competency in procedures such as paracentesis for certification. ${ }^{12}$ However, the Accreditation Council for Graduate Medical Education (ACGME) requirements state that internal medicine programs "must offer residents the opportunity to demonstrate competence" in the performance of procedures such as paracentesis, thoracentesis, and central venous catheter insertion. ${ }^{13}$ The American Board of Family Medicine (ABFM) 
does not outline specific procedural competence for initial certification. ${ }^{14}$ The ACGME states that family medicine residents "must receive training to perform those clinical procedures required for their future practices" but allows each program to determine which procedures to require. ${ }^{15}$ Due to this uncertainty, practicing hospitalists are likely to have variable training and competence in bedside procedures such as paracentesis.

We previously showed that internal medicine residents rotating on the hepatology service of an academic medical center performed $59 \%$ of paracentesis procedures at the bedside. ${ }^{16}$ These findings are in contrast to national data showing that $74 \%$ of paracentesis procedures performed on Medicare beneficiaries were performed by radiologists. ${ }^{7}$ Practice patterns at university hospitals may not be reflected in this data because the study was limited to Medicare beneficiaries and included ambulatory patients. ${ }^{7}$ In addition to uncertainty about who is performing this procedure in inpatient settings, little is known about the effect of specialty on postparacentesis clinical outcomes. ${ }^{16,17}$

The current study had 3 aims: (1) evaluate which clinical specialties perform paracentesis procedures at university hospitals; (2) model patient characteristics associated with procedures performed at the bedside versus those referred to IR; and (3) among patients with a similar likelihood of IR referral, evaluate length of stay (LOS) and hospital costs of patients undergoing procedures performed by different specialties.

\section{METHODS}

We performed an observational administrative database review of patients who underwent paracentesis procedures in hospitals participating in the University HealthSystem Consortium (UHC) Clinical Database from January 2010 through December 2012. UHC is an alliance of 120 nonprofit academic medical centers and their 290 affiliated hospitals. UHC maintains databases containing clinical, operational, financial, and patient safety data from affiliated hospitals. Using the UHC database, we described the characteristics of all patients who underwent paracentesis procedures by clinical specialty performing the procedure. We then modeled the effects of patient characteristics on decision-making about IR referral. Finally, among patients with a homogeneous predicted probability of IR referral, we compared LOS and direct costs by specialty performing the procedure. The Northwestern University institutional review board approved this study.

\section{Procedure}

We queried the UHC database for all patients over the age of 18 years who underwent paracentesis procedures (International Classification of Disease Revision 9 [ICD-9] procedure code 54.91) and had at least
1 diagnosis code of liver disease (571.x). We excluded patients admitted to obstetrics. The query included patient and clinical characteristics such as admission, discharge, and procedure date; age, gender, procedure provider specialty, and intensive care unit (ICU) stay. We also obtained all ICD-9 codes associated with the admission including obesity, severe liver disease, coagulation disorders, blood loss anemia, hyponatremia, hypotension, thrombocytopenia, liver transplant before or during the admission, awaiting liver transplant, and complications of liver transplant. We used ICD-9 codes to calculate patients' Charlson score ${ }^{18,19}$ to assess severity of illness on admission.

LOS and total direct hospital costs were compared among patients with a paracentesis performed by a single clinical group and among patients with a similar predicted probability of IR referral. UHC generates direct cost estimates by applying Medicare Cost Report ratios of cost to charges with the labor cost further adjusted by the respective area wage index. Hospital costs were not available from $8.3 \%$ of UHC hospitals. We therefore based cost estimates on nonmissing data.

Paracentesis provider specialties were divided into 6 general categories: (1) IR (interventional and diagnostic radiology); (2) medicine (family medicine, general medicine, and hospital medicine); (3) subspecialty medicine (infectious disease, cardiology, nephrology, hematology/oncology, endocrinology, pulmonary, and geriatrics); (4) gastroenterology/hepatology (gastroenterology, hepatology, and transplant medicine); (5) general surgery (general surgery and transplant surgery); and (6) all other (included unclassified specialties). We present patient characteristics categorized by these specialty groups and for admissions in which multiple specialties performed procedures.

\section{Study Design}

To analyze an individual patient's likelihood of IR referral, we needed to restrict our sample to discharges where only 1 clinical specialty performed a paracentesis. Therefore, we excluded hybrid discharges with procedures performed by more than 1 specialty in a single admission as well as discharges with procedures performed by all other specialties. To compare LOS and direct cost outcomes, and to minimize selection bias among exclusively IR-treated patients, we excluded hospitals without procedures done by both IR and medicine.

We modeled referral to IR as a function of patients' demographic and clinical variables, which we believed would affect the probability of referral. We then examined the IR referral model predicted probabilities (propensity score). ${ }^{20}$ Finally, we examined mean differences in LOS and direct costs among discharges with a single clinical specialty group, while using the predicted probability of referral as a filter to compare these outcomes by specialty. We further tested 
specialty differences in LOS and direct costs controlling for demographic and clinical variables.

\section{Statistical Analysis}

To test the significance of differences between demographic and clinical characteristics of patients across specialties, we used $\chi^{2}$ tests for categorical variables and analysis of variance or the Kruskal-Wallis rank test for continuous variables. Random effects logistic regression, which adjusts standard errors for clustering by hospital, was used to model the likelihood of referral to IR. Independent variables included patient age, gender, obesity, coagulation disorders, blood loss anemia, hyponatremia, hypotension, thrombocytopenia, liver transplant before hospitalization, liver transplant during hospitalization, awaiting transplant, complications of liver transplant, ICU stay, Charlson score, and number of paracentesis procedures performed during the admission. Predicted probabilities derived from this IR referral model were used to investigate selection bias in our subsequent analyses of LOS and costs. ${ }^{20}$

We used random effects multiple linear regression to test the association of procedure specialty with hospital LOS and total direct costs, controlling for the same independent variables listed above. Analyses were conducted using both actual LOS in days and Medicare costs. We also performed a log transformation of LOS and costs to account for rightward skew. We only present actual LOS and cost results because results were virtually identical. We used SAS version 9 (SAS Institute Inc., Cary, NC) to extract data from the UHC Clinical Database. We performed all statistical analyses using Stata version 12 (StataCorp LP, College Station, TX).

\section{RESULTS}

\section{Procedure and Discharge Level Results}

There were 97,577 paracentesis procedures performed during 70,862 hospital admissions in 204 UHC hospitals during the study period. Table 1 shows specific specialty groups for each procedure. The all other category consisted of 17,558 subspecialty groups including 9,434 with specialty unknown. Twenty-nine percent of procedures were performed in IR versus $27 \%$ by medicine, $11 \%$ by gastroenterology/hepatology, and $11 \%$ by subspecialty medicine.

Table 2 presents patient characteristics for 70,862 hospital discharges with paracentesis procedures grouped by whether single or multiple specialties performed procedures. Patient characteristics were significantly different across specialty groups. Medicine, subspecialty medicine, and gastroenterology/hepatology patients were younger, more likely to be male, and more likely to have severe liver disease, coagulation disorders, hypotension, and hyponatremia than IR patients.

\section{IR Referral Model}

We first excluded 6030/70,862 discharges (8.5\%) from 59 hospitals without both IR and medicine pro-
TABLE 1. Group Frequencies of 97,577 Paracentesis Procedures by Specialty Within Specialty Groups

\begin{tabular}{lcr}
\hline Specialty Group & No. & $\%$ \\
\hline Interventional radiology & 28,414 & 29.1 \\
Medicine & 26,031 & 26.7 \\
Family medicine & 1,026 & 1.1 \\
General medicine & 21,787 & 22.3 \\
Hospitalist & 3,218 & 3.3 \\
Subspecialty medicine & 10,558 & 10.8 \\
Infectious disease & 848 & 0.9 \\
Nephrology & 615 & 0.6 \\
Cardiology & 991 & 1.0 \\
Hematology oncology & 795 & 0.8 \\
Endocrinology & 359 & 0.4 \\
Pulmonology & 6,605 & 6.8 \\
Geriatics & 345 & 0.4 \\
Gastroenterologylhepatology & 11,143 & 11.4 \\
Transplant medicine & 99 & 0.1 \\
Hepatalogy & 874 & 0.9 \\
Gastroenterology & 10,170 & 10.4 \\
General surgery & 3,873 & 4.0 \\
Transplant surgery & 2,146 & 2.2 \\
General surgery & 1,727 & 1.8 \\
All other & 17,558 & 18.0 \\
Specialty unknown & 9,434 & 9.7 \\
\hline
\end{tabular}

cedures. We then further excluded 24,986/70,862 $(35.3 \%)$ discharges with procedures performed by multiple specialties during the same admission. Finally, we excluded 5555/70,862 (7.8\%) of discharges with procedure specialty coded as all other. Therefore, 34,291 (48.4\%) discharges $(43,337 /$ 97,$577 ; 44.4 \%$ procedures) from 145 UHC hospitals with paracentesis procedures performed by a single clinical specialty group remained for the IR referral analysis sample. Among admissions with multiple specialty paracentesis performed within the same admission, 3128/26,606 admissions with any IR procedure $(11.8 \%)$ had a different specialty ascribed to the first, second, or third paracentesis with a subsequent IR procedure.

Model results (Table 3) indicate that patients who were obese (odds ratio [OR]: $1.25 ; 95 \%$ confidence interval [CI]: $1.10-1.43)$ or had a liver transplant on a prior admission (OR: 2.03; 95\% CI: 1.40-2.95) were more likely to be referred to IR. However, male patients (OR: 0.89 ; 95\% CI: $0.83-0.95$ ), or patients who required an ICU stay (OR: 0.39 ; 95\% CI: $0.36-$ 0.43 ) were less likely to have IR procedures. Other patient factors reducing the likelihood of IR referral included characteristics associated with higher severity of illness (coagulation disorders, hyponatremia, hypotension, and thrombocytopenia).

\section{Predicted Probabilities of IR Referral}

Figure 1 presents the distribution of predicted probabilities for IR referral. Predicted probabilities were low overall, with very few patients having an equal 
TABLE 2. Characteristics of Patients Discharged by Medical Specialty Groups Performing Paracentesis Procedures at University HealthSystem Consortium Hospitals ( $N=70,862$ Discharges From 2010-2012 in 204 Hospitals)

\begin{tabular}{|c|c|c|c|c|c|c|c|c|}
\hline & $\begin{array}{c}\text { All Discharges, } \\
\mathrm{N}=70,862\end{array}$ & $\begin{array}{c}\text { Interventional } \\
\text { Radiology, } \\
n=9,348\end{array}$ & $\begin{array}{l}\text { Medicine, } \\
n=13,789\end{array}$ & $\begin{array}{l}\text { Subspecialty } \\
\text { Medicine, } \\
n=5,085\end{array}$ & $\begin{array}{c}\text { Gastroenterology/ } \\
\text { Hepatology, } \\
n=6,664\end{array}$ & $\begin{array}{l}\text { General Surgery, } \\
\quad n=1,891\end{array}$ & $\begin{array}{l}\text { All Other, } \\
n=7,912\end{array}$ & $\begin{array}{c}\text { Discharges } \\
\text { With Multiple } \\
\text { Specialties, } \\
n=26,173\end{array}$ \\
\hline \multicolumn{9}{|l|}{ Age group, y (\%) } \\
\hline $18-49$ & 25.4 & 22.5 & 27.6 & 24.9 & 23.5 & 20.8 & 25.5 & 26.1 \\
\hline $50-59$ & 39.8 & 39.8 & 40.9 & 39.4 & 41.5 & 40.3 & 40.0 & 38.7 \\
\hline $60-69$ & 24.7 & 24.9 & 21.6 & 24.7 & 26.5 & 30.0 & 23.6 & 25.8 \\
\hline $70+$ & 10.1 & 12.9 & 9.9 & 11.1 & 8.4 & 8.9 & 11.0 & 9.4 \\
\hline Male (\%) & 65.5 & 64.2 & 67.6 & 67.5 & 65.7 & 66.6 & 65.7 & 64.2 \\
\hline Severe liver disease (\%)* & 73.7 & 65.3 & 67.8 & 71.0 & 75.3 & 66.6 & 67.6 & 82.1 \\
\hline Obesity (BMI 40+) (\%) & 6.3 & 6.1 & 5.3 & 5.7 & 5.1 & 5.8 & 5.2 & 7.6 \\
\hline Any intensive care unit stay (\%) & 31.0 & 10.9 & 16.8 & 50.5 & 16.9 & 36.7 & 22.3 & 47.8 \\
\hline Coagulation disorders $(\%)$ & 24.3 & 14.8 & 20.2 & 29.9 & 16.1 & 19.0 & 17.8 & 33.1 \\
\hline Blood loss anemia $(\%)$ & 3.4 & 1.3 & 2.8 & 2.7 & 2.7 & 1.9 & 2.1 & 5.2 \\
\hline Hyponatremia $(\%)$ & 29.9 & 27.1 & 29.2 & 28.9 & 28.0 & 26.6 & 27.3 & 33.1 \\
\hline Hypotension (\%) & 9.8 & 7.0 & 8.0 & 11.0 & 7.7 & 10.5 & 8.1 & 12.4 \\
\hline Thrombocytopenia (\%) & 29.6 & 24.6 & 28.3 & 32.5 & 22.1 & 21.5 & 24.0 & 35.8 \\
\hline Complication of transplant (\%) & 3.3 & 2.1 & 1.1 & 2.4 & 4.0 & 10.3 & 2.7 & 4.7 \\
\hline Awaiting liver transplant (\%) & 7.6 & 6.4 & 4.0 & 5.4 & 12.8 & 16.0 & 7.8 & 8.2 \\
\hline Prior liver transplant (\%) & 0.5 & 0.8 & 0.3 & 0.3 & 0.7 & 0.7 & 0.4 & 0.6 \\
\hline Liver transplant procedure (\%) & 2.7 & 0.0 & 0.0 & 0.3 & 0.4 & 15.6 & 1.6 & 5.6 \\
\hline Mean Charlson score (SD) & $4.51(2.17)$ & $4.28(2.26)$ & $4.16(2.17)$ & $4.72(2.30)$ & $4.30(1.98)$ & $4.26(2.22)$ & $4.36(2.30)$ & $4.84(2.07)$ \\
\hline Mean paracentesis procedures per discharge (SD) & $1.38(0.88)$ & $1.21(0.56)$ & $1.26(0.66)$ & $1.30(0.76)$ & $1.31(0.70)$ & $1.28(0.78)$ & $1.22(0.61)$ & $1.58(1.13)$ \\
\hline
\end{tabular}

NOTE: All patient characteristic comparisons across all specialty groups, $P<0.0001$. Abbreviations: BMI, body mass index; $\mathrm{SD}$, standard deviation.

chance of referral-the standard often used in comparative effectiveness analyses from observational data. Figure 1 indicates that IR referral probabilities were clustered in an unusual bimodal distribution.

\section{TABLE 3. Random Effects Logistic Regression Model of Likelihood of Interventional Radiology Paracentesis ( $\mathrm{N}=34,291$ Discharges From 145 Hospitals)}

\begin{tabular}{lccc}
\hline & & \multicolumn{2}{c}{$95 \% \mathrm{Cl}$} \\
\cline { 4 - 4 } & Odds Ratio & Lower & Upper \\
\hline Age group, y & & & \\
$18-49$ & Reference & & \\
$50-59$ & 1.05 & 0.97 & 1.14 \\
$60-69$ & 1.12 & 1.02 & 1.22 \\
$70+$ & 1.11 & 0.99 & 1.24 \\
Male & 0.89 & 0.83 & 0.95 \\
Obesity, BMI 40+ & 1.25 & 1.10 & 1.43 \\
ICU care & 0.39 & 0.36 & 0.43 \\
Coagulation disorders & 0.68 & 0.63 & 0.75 \\
Blood loss anemia & 0.52 & 0.41 & 0.66 \\
Hyponatremia & 0.85 & 0.80 & 0.92 \\
Hypotension & 0.83 & 0.74 & 0.93 \\
Thrombocytopenia & 0.94 & 0.87 & 1.01 \\
Prior liver transplant & 0.08 & 0.03 & 0.23 \\
Awaiting liver transplant & 0.86 & 0.76 & 0.98 \\
Complication of liver transplant & 1.07 & 0.88 & 1.31 \\
Liver transplant procedure & 2.03 & 1.40 & 2.95 \\
Charlson score & 1.00 & 0.99 & 1.01 \\
Number of paracentesis procedures & 0.90 & 0.85 & 0.95 \\
\hline
\end{tabular}

NOTE: Abbreviations: BMI, body mass index; Cl, confidence interval; ICU, intensive care unit.
The cluster on the left, which centers around a $15 \%$ predicted probability of IR referral, consists of discharges with patient characteristics that were associated with a very low chance of an IR paracentesis. We therefore used this distribution to conduct comparative analyses of admission outcomes between clinical specialty groups, choosing to examine patients with a $20 \%$ or greater chance of IR referral.

Post hoc analysis revealed that the biggest factor driving low predicted probability of IR referral was whether patients experienced an ICU stay at any time

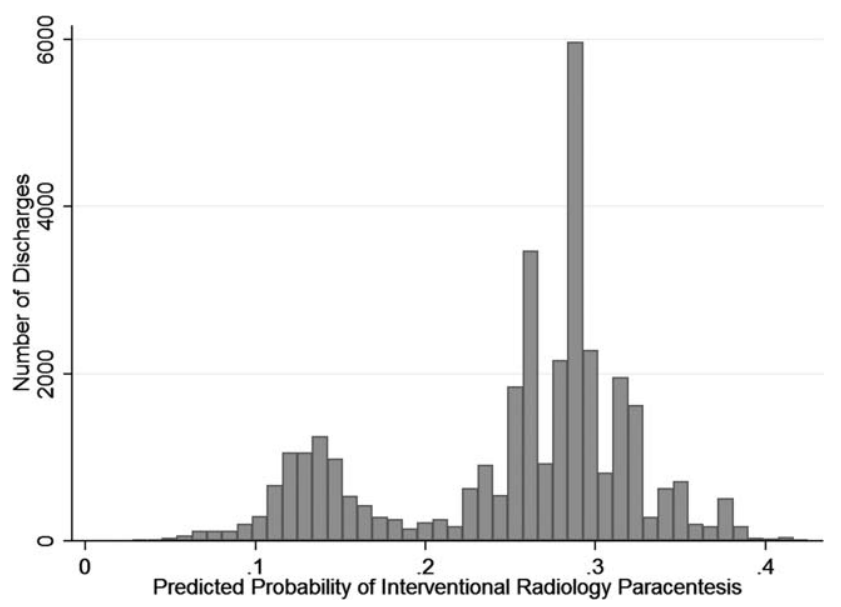

FIG. 1. Distribution of predicted probability of interventional radiology paracentesis. Discharges with paracentesis procedures from 145 University HealthSystem Consortium hospitals performed by a single specialty group $(n=34,291)$. 
TABLE 4. Length of Stay and Total Hospital Direct Costs for Paracentesis Procedure Discharges Performed by a Single Specialty Group (Interventional Radiology Referral Probability $\geq 0.2$ )

\begin{tabular}{|c|c|c|c|c|c|c|}
\hline & $\begin{array}{c}\text { All Admissions } \\
n=26,615\end{array}$ & $\begin{array}{l}\text { Interventional Radiology } \\
\qquad \mathrm{n}=7,677\end{array}$ & $\begin{array}{c}\text { Medicine } \\
n=10,413\end{array}$ & $\begin{array}{l}\text { Medicine Subspecialties } \\
\qquad n=2,210\end{array}$ & $\begin{array}{l}\text { Gastroenterology/ Hepatology } \\
\qquad n=5,182\end{array}$ & $\begin{array}{c}\text { General Surgery } \\
n=1,133\end{array}$ \\
\hline \multirow[t]{2}{*}{ Mean length of stay, d (SD) } & $5.57(5.63)$ & $5.20(4.72)$ & $5.59(5.85)$ & $6.28(6.47)$ & $5.54(5.31)$ & $6.67(8.16)$ \\
\hline & $\begin{array}{c}\text { All Admissions } \\
n=24,408\end{array}$ & $\begin{array}{l}\text { Interventional Radiology } \\
n=7,265\end{array}$ & $\begin{array}{l}\text { Medicine } \\
\mathrm{n}=8,965\end{array}$ & $\begin{array}{l}\text { Medicine Subspecialties } \\
\qquad n=2,064\end{array}$ & $\begin{array}{l}\text { Gastroenterology/Hepatology } \\
\qquad n=5,031\end{array}$ & $\begin{array}{c}\text { General Surgery } \\
n=1,083\end{array}$ \\
\hline Mean total direct cost, \$ (SD)* & $11,447(12,247)$ & $10,975(9,723)$ & $10,517(10,895)$ & $13,705(16,591)$ & $12,000(11,712)$ & $15,448(23,807)$ \\
\hline
\end{tabular}

NOTE: Length of stay and direct cost comparisons across all specialty groups, $P<0.0001$. Abbreviations: $S D$, standard deviation. Data not adjusted for patient characteristics.

${ }^{*}$ Total costs $n=8.3 \%$ missing.

during hospitalization. Among the discharges with a predicted probability $\geq 0.2 \quad(\mathrm{n}=26,615$ discharges $)$, there were only 87 discharges with ICU stays $(0.3 \%)$. For the discharges with predicted probability $<0.2$ $(\mathrm{n}=7676), 91.9 \%(\mathrm{n}=7055)$ had an ICU admission. We therefore used a threshold of 0.2 or greater to present the most comparable LOS and direct cost differences.

\section{LOS and Cost Comparisons by Specialty}

Mean LOS and hospital direct costs by specialty for our final analysis sample can be found in Table 4; differences between specialties were significant $(P<0.0001)$. Patients undergoing IR procedures had equivalent LOS and costs to medicine patients, but lower LOS and costs than other clinical specialty groups. Random effects linear regression showed that neither medicine nor gastroenterology/hepatology patients had significantly different LOS from IR patients, but subspecialty medicine was associated with 0.89 additional days and general surgery with 1.47 additional days (both $P<0.0001 ; R^{2}=0.10$ ). In the direct cost regression model, medicine patients were associated with $\$ 1308$ lower costs and gastroenterology/hepatology patients with $\$ 803$ lower costs than IR patients (both $P=0.0001$ ), whereas subspecialty medicine and general surgery had higher direct costs per discharge of $\$ 1886$ and $\$ 3039$, respectively (both $P<0.0001, R^{2}=0.19$ ). Older age, obesity, coagulopathy, hyponatremia, hypotension, thrombocytopenia, liver transplant status, ICU care, higher Charlson score, and higher number of paracentesis procedures performed were all significantly associated with higher LOS and hospital costs in these linear models.

\section{DISCUSSION}

This study showed that internal medicine- and family medicine-trained clinicians perform approximately half of the inpatient paracentesis procedures at university hospitals and their affiliates. This confirms findings from our earlier single-institution study ${ }^{16}$ but contrasts with previously published reports involving
Medicare data. The earlier report, using Medicare claims and including ambulatory procedures, revealed that primary care physicians and gastroenterologists only performed approximately $10 \%$ of US paracentesis procedures in 2008. ${ }^{7}$ Our findings suggest that practices are different at university hospitals, where patients with severe liver disease often seek care. Because we used the UHC database, it was not possible to determine if the clinicians who performed paracentesis procedures in this study were internal medicine or family medicine residents, fellows, or attending physicians. However, findings from our own institution show that the vast majority of bedside paracentesis procedures are performed by internal medicine residents. ${ }^{16}$

Our findings have implications for certification of internal medicine and family medicine trainees. In 2008, the ABIM removed the requirement that internal medicine residents demonstrate competency in paracentesis. ${ }^{12}$ This decision was informed by a lack of standardized methods to determine procedural competency and published surveys showing that internal medicine and hospitalist physicians rarely performed bedside procedures. ${ }^{5,6}$ Despite this policy change, our findings show that current clinical practice at university hospitals does not reflect national practice patterns or certification requirements, because many internal medicine- and family medicine-trained clinicians still perform paracentesis procedures. This is concerning because internal medicine and family medicine trainees report variable confidence, experience, expertise, and supervision regarding performance of invasive procedures. ${ }^{9,10,21-24}$ Furthermore, earlier research also demonstrates that graduating residents and fellows are not able to competently perform common bedside procedures such as thoracentesis, temporary hemodialysis catheter insertion, and lumbar puncture. ${ }^{25-27}$

The American Association for the Study of Liver Diseases (AASLD) recommends that trained clinicians perform paracentesis procedures. ${ }^{3,4}$ However, the AASLD provides no definition for how training should occur. Because competency in this procedure is not specifically required by the ABIM, ABFM, or 
ACGME, a paradoxical situation occurs in which internal medicine and family medicine residents, and internal medicine-trained fellows and faculty continue to perform paracentesis procedures on highly complex patients, but are no longer required to be competent to do so.

In earlier research we showed that simulation-based mastery learning (SBML) was an effective method to boost internal medicine residents' paracentesis skills. ${ }^{28}$ In SBML, all trainees must meet or exceed a minimum passing score on a simulated procedure before performing one on an actual patient. ${ }^{29}$ This approach improves clinical care and outcomes in procedures such as central venous catheter insertion ${ }^{30,31}$ and advanced cardiac life support. ${ }^{32}$ SBML-trained residents also performed safe paracentesis procedures with shorter hospital LOS, fewer ICU transfers, and fewer blood product transfusions than IR procedures. ${ }^{16}$ Based on the results of this study, AASLD guidelines regarding training, and our experience with SBML, we recommend that all clinicians complete paracentesis SBML training before performing procedures on patients.

Using our propensity model we identified patient characteristics that were associated with IR referral. Patients with a liver transplant were more likely to be cared for in IR. This may be due to a belief that postoperative procedures are anatomically more complex or because surgical trainees do not commonly perform this procedure. The current study confirms findings from earlier work that obese and female patients are more likely to be referred to IR. ${ }^{16}$ IR referral of obese patients is likely to occur because paracentesis procedures are technically more difficult. We have no explanation why female patients were more likely to be referred to IR, because most decisions appear to be discretionary. Prospective studies are needed to determine evidence-based recommendations regarding paracentesis procedure location. Patients with more comorbidities (eg, ICU stay, awaiting liver transplant, coagulation disorders) were more likely to undergo bedside procedures. The complexity of patients undergoing bedside paracentesis procedures reinforces the need for rigorous skill assessment for clinicians who perform them because complications such as intraperitoneal bleeding can be fatal.

Finally, we showed that LOS was similar but hospital direct costs were $\$ 800$ to $\$ 1300$ lower for patients whose paracentesis procedure was performed by medicine or gastroenterology/hepatology compared to IR. Medical subspecialties and surgery procedures were more expensive than IR, consistent with the higher LOS seen in these groups. IR procedures add costs due to facility charges for space, personnel, and equipment. ${ }^{33}$ At our institution, the hospital cost of an IR paracentesis in 2012 was $\$ 361$. If we use this figure, and assume costs are similar across university hospitals, the resultant cost savings would be $\$ 10,257,454$ (for the procedure alone) if all procedures referred to
IR in this 2-year study were instead performed at the bedside. This estimate is approximate because it does not consider factors such as cost of clinician staffing models, which may differ across UHC hospitals. As hospitals look to reduce costs, potential savings due to appropriate use of bedside and IR procedures should be considered. This is especially important because there is no evidence that the extra expense of IR procedures is justified due to improved patient outcomes.

This study has several limitations. First, this was an observational study. Although the database was large, we were limited by coding accuracy and could not control for all potential confounding factors such as Model for End-Stage Liver Disease score, ${ }^{34,35}$ other specific laboratory values, amount of ascites fluid removed, or bedside procedure failures later referred to IR. However, we do know that only a small number of second, third, or fourth procedures were subsequently referred to IR after earlier ones were performed at the bedside. Additionally the UHC database does not include patient-specific data, and therefore we could not adjust for multiple visits or procedures by the same patient. Second, we were unable to determine the level of teaching involvement at each UHC affiliated hospital. Community hospitals where attendings managed most of the patients without trainees could not be differentiated from university hospitals where trainees were involved in most patients' care. Third, we did not have specialty information for 9434 $(9.7 \%)$ procedures and had to exclude these cases. We also excluded a large number of paracentesis procedures in our final outcomes analysis. However, this was necessary because we needed to perform a patient-level analysis to ensure the propensity and outcomes models were accurate. Finally, we did not evaluate inpatient mortality or 30-day hospital readmission rates. Mortality and readmission from complications of a paracentesis procedure are rare events. ${ }^{3,4,36}$ However, mortality and hospital readmission among patients with liver disease are relatively common. ${ }^{37,38}$ It was impossible to link these outcomes to a paracentesis procedure without the ability to perform medical records review.

In conclusion, paracentesis procedures are performed frequently by internal medicine- and family medicinetrained clinicians in university hospitals. Because of these findings regarding current practice patterns, we believe the ACGME, ABIM, and ABFM should clarify their policies to require that residents are competent to perform paracentesis procedures before performing them on patients. This may improve supervision and training for paracentesis procedures that are already occurring and possibly encourage performance of additional, less costly bedside procedures.

\section{Acknowledgements}

The authors acknowledge Drs. Douglas Vaughan and Mark Williams for their support and encouragement of this work.

Disclosure: Nothing to report. 


\section{References}

1. Runyon BA. A primer on detecting cirrhosis and caring for these patients without causing harm. Int J Hepatol. 2011:801983.

2. Lefton HB, Rosa A, Cohen M. Diagnosis and epidemiology of cirrhosis. Med Clin North Am. 2009;93(4):787-799.

3. Runyon BA; AASLD Practice Guidelines Committee. Management of adult patients with ascites due to cirrhosis: an update. Hepatology. 2009;49(6):2087-2107.

4. Runyan BA; AASLD Practice Guidelines Committee. Management of adult patients with ascites due to cirrhosis: update 2012. Hepatology. 2009;49:2087-2107. Available at: http://www.aasld.org/practiceguidelines/Documents/ascitesupdate2013.pdf. Accessed October 21, 2013.

5. Thakkar R, Wright SM, Alguire P, Wigton RS, Boonyasai RT. Procedures performed by hospitalist and non-hospitalist general internists. J Gen Intern Med. 2010;25(5):448-452.

6. Wigton RS, Alguire P. The declining number and variety of procedures done by general internists: a resurvey of members of the American College of Physicians. Ann Intern Med. 2007:146(5):355-360.

7. Duszak R Jr, Chatterjee AR, Schneider DA. National fluid shifts: fifteen-year trends in paracentesis and thoracentesis procedures. $J \mathrm{Am}$ Coll Radiol. 2010;7(11):859-864.

8. Duffy FD, Holmboe ES. What procedures should internists do? Ann Intern Med. 2007;146(5):392-393.

9. Huang GC, Smith CC, Gordon CE, Feller-Kopman DJ, Davis RB, Phillips RS. Beyond the comfort zone: residents assess their comfort performing inpatient medicine procedures. Am J Med. 2006; 119(1)71.e17-e24.

10. Sharp LK, Wang R, Lipsky MS. Perception of competency to perform procedures and future practice intent: a national survey of family practice residents. Acad Med. 2003;78(9):926-932.

11. Guardino JM, Proctor DD, Lopez R, Carey W. Utilization of and adherence to the gastroenterology core curriculum on hepatology training during a gastrointestinal fellowship. Clin Gastroenterol Hepatol. 2008;6(6):682-688.

12. American Board of Internal Medicine. Internal medicine policies. Available at: http://www.abim.org/certification/policies/imss/im.aspx. Accessed December 21, 2013.

13. ACGME program requirements for graduate medical education in internal medicine. Available at: http://acgme.org/acgmeweb/portals/0/ PFassets/2013-PR-FAQ-PIF/140_internal_medicine_07012013.pdf. Accessed December 17, 2013.

14. American Board of Family Medicine residency requirements. Available at: https://www.theabfm.org/cert/guidelines.aspx. Accessed December 17, 2013.

15. ACGME program requirements for graduate medical education in family medicine. Available at: http://www.acgme.org/acgmeweb/Portals/0/PFAssets/ProgramRequirements/120pr07012007.pdf. Accessed December 17, 2013.

16. Barsuk JH, Cohen ER, Feinglass J, McGaghie WC, Wayne DB. Clinical outcomes after bedside and interventional radiology paracentesis procedures. Am J Med. 2013;126(4):349-356.

17. Grabau CM, Crago SF, Hoff LK, et al. Performance standards for therapeutic abdominal paracentesis. Hepatology. 2004;40(2):484488.

18. Deyo RA, Cherkin DC, Ciol MA. Adapting a clinical comorbidity index for use with ICD-9-CM administrative databases. J Clin Epidemiol. 1992;45(6):613-619.

19. Romano PS, Roos LL, Jollis JG. Adapting a clinical comorbidity index for use with ICD-9-CM administrative data: differing perspectives. J Clin Epidemiol. 1993;46(10):1075-1079; discussion 1081-1090.
20. Rosenbaum PR. The role of known effects in observational studies. Biometrics. 1998;45(2):557-569.

21. Farnan JM, Petty LA, Georgitis E, et al. A systematic review: the effect of clinical supervision on patient and residency education outcomes. Acad Med. 2012;87(4):428-442.

22. Lucas BP, Asbury JK, Wang Y, et al. Impact of a bedside procedure service on general medicine inpatients: a firm-based trial. J Hosp Med. 2007;2(3):143-149.

23. Haber LA, Lau CY, Sharpe BA, Arora VM, Farnan JM, Ranji SR. Effects of increased overnight supervision on resident education, decision-making, and autonomy. J Hosp Med. 2012;7(8):606-610.

24. Berns JS, O’Neill WC. Performance of procedures by nephrologists and nephrology fellows at U.S. nephrology training programs. Clin J Am Soc Nephrol. 2008;3(4):941-947.

25. Wayne DB, Barsuk JH, O'Leary K, Fudala M, McGaghie WC. Mastery learning of thoracentesis skills by internal medicine residents using simulation technology and deliberate practice. J Hosp Med. 2008;3:49-54.

26. Barsuk JH, Ahya SN, Cohen ER, McGaghie WC, Wayne DB. Mastery learning of temporary dialysis catheter insertion skills by nephrology fellows using simulation technology and deliberate practice. Am J Kidney Dis. 2009;54:70-76.

27. Barsuk JH, Cohen ER, Caprio T, McGaghie WC, Simuni T, Wayne DB. Simulation-based education with mastery learning improves residents' lumbar puncture skills. Neurology. 2012;79:132-137.

28. Barsuk JH, Cohen ER, Vozenilek JA, O'Connor LM, McGaghie WC, Wayne DB. Simulation-based education with mastery learning improves paracentesis skills. J Grad Med Educ. 2012;4(1):23-27.

29. McGaghie WC, Issenberg SB, Cohen ER, Barsuk JH, Wayne DB. Medical education featuring mastery learning with deliberate practice can lead to better health for individuals and populations. Acad Med. 2011; 86(11):e8-e9.

30. Barsuk JH, McGaghie WC, Cohen ER, O'Leary KJ, Wayne DB. Simulation-based mastery learning reduces complications during central venous catheter insertion in a medical intensive care unit. Crit Care Med. 2009;37(10):2697-2701.

31. Barsuk JH, Cohen ER, Feinglass J, McGaghie WC, Wayne DB. Use of simulation-based education to reduce catheter-related bloodstream infections. Arch Intern Med. 2009;169(15):1420-1423.

32. Didwania A, McGaghie WC, Cohen ER, et al. Progress toward improving the quality of cardiac arrest medical team responses at an academic teaching hospital. J Grad Med Educ. 2011;3(2):211-216.

33. Saini S, Seltzer SE, Bramson RT, et al. Technical cost of radiologic examinations: analysis across imaging modalities. Radiology. 2000; 216(1):269-272.

34. Kamath PS, Wiesner RH, Malinchoc M, et al. A model to predict survival in patients with end-stage liver disease. Hepatology. 2001;33(2): 464-470.

35. Wiesner R, Edwards E, Freeman R, et al. Model for end-stage liver disease (MELD) and allocation of donor livers. Gastroenterology. 2003;124(1):91-96.

36. Thomsen TW, Shaffer RW, White B, Setnik GS. Videos in clinical medicine. Paracentesis. N Engl J Med. 2006;355(19):e21.

37. Center for Disease Control and Prevention. Chronic liver disease or cirrhosis. National Hospital Discharge Survey: 2010 detailed diagnosis and procedure tables, number of first-listed diagnoses (see ICD9CM code 571). Available at: http://www.cdc.gov/nchs/fastats/liverdis.htm. Accessed October 19, 2013.

38. Berman K, Tandra S, Forssell K, et al. Incidence and predictors of 30day readmission among patients hospitalized for advanced liver disease. Clin Gastroenterol Hepatol. 2011;9(3):254-259. 